\title{
La mise en scène des pièces de théâtre européennes par Ali Raffi en Iran et Circulation des savoirs entre la France et l'Iran
}

\author{
Mojgan Mahdavi Zadeh \\ Université d'Ispahan
}

\section{1) Introduction}

L'élément le plus dominant des théâtres égyptien, grec, romain, et persan était leur caractère religieux. L'univers dramatique persan s'appuyait sur les légendes et les mythes, mais toujours dans un contexte rituel. Des traces de diverses cérémonies, telles les cérémonies d'initiation au culte de Dieu Mithra ou celles accompagnées de danses, en l'honneur de la Déesse Ânâhitân ${ }^{1}$ datant de l'Antiquité, prouvent la fertilité de l'histoire du théâtre persan.

Plus tard, en particulier suite à l'illustration du Livre des Rois ${ }^{2}$, la plus grande épopée persane, par Ferdowsi ${ }^{3}$, des conteurs se sont mis à réaliser des récits à épisodes, des histoires épiques, de divers romans picaresques, et des récitations des morceaux choisis des ouvrages sur des places publiques.

Il est à noter que certaines religions monothéistes ne s'adaptaient pas facilement à la mise en scène de Dieu et des personnages sacrés, puisqu'elles pensaient qu'aucune personne ordinaire n'est capable d'assumer une telle divinité. C'est pourquoi elles interdisaient la danse, la musique de caractère non religieux et l'incarnation de l'Esprit divin dans un personnage intitulé «acteur » lors d'une réalisation théâtrale. Alors que pour les pays polythéistes tels l'Inde, la Chine, et la Grèce, lors de la réalisation sur scène d'une pièce, chaque dieu, prophète ou maître, pouvait facilement être incarné.

\footnotetext{
1 Ânâhitâ, Nâhid, Anaïd ou Anâhïd est une ancienne divinité perse assimilable à la déesse sémitique Ishtar et à la déesse-rivière indienne Sarasvatî.

${ }^{2}$ Le Livre des Rois contient 50000 vers qui incarnent les origines de la Perse Antique, la culture et la civilisation de ce pays. À l'heure actuelle, ce trésor appartient au patrimoine culturel commun de tout peuple persanophone.

${ }^{3}$ Ferdowsi est le poète iranien du $\mathrm{X}^{\text {ème }}$ siècle qui a contribué à la survie de la langue persane. Il est à noter qu'une avenue située dans le Parc Monceau à Paris porte le nom de ce grand poète iranien depuis 1935.
} 
Depuis le $\mathrm{XIX}^{\mathrm{e}}$ siècle et suite à la spécialisation des étudiants envoyés en Europe, le théâtre moderne iranien d'inspiration occidentale a eu l'occasion de s'épanouir. Parmi ces diplômés figure Ali Raffi, docteur en Études théâtrales de l'Université de la Sorbonne-Nouvelle. Privilégiant la couleur, le mouvement et l'intuition depuis 1974, Raffi était spécialiste des adaptations théâtrales. Il a su anoblir et enrichir la mentalité du public et a eu une influence considérable sur la culture iranienne par le biais de ses mises en scène de pièces de théâtre françaises, ainsi que de ses représentations à la française; ce qui a abouti au développement de l'imaginaire des pièces.

La problématique qui motive la rédaction de cet article consiste à découvrir les échanges interculturels franco-iraniens qui ont été mis à œuvre par Ali Raffi, grâce à la mise en scène des pièces de théâtre françaises ou à la française en Iran. Nous allons également vérifier jusqu'à quel point ces échanges culturels et artistiques ont fourni de nouveaux horizons dans la voie de l'épanouissement spirituel d'un peuple. Le but de ce travail est la mise en relief des conséquences issues de la mise en scène des pièces de théâtre des grands auteurs européens et en particulier français en Iran. Nous nous intéressons également aux raisons pour lesquelles les élites contemporaines iraniennes s'attachent beaucoup à ces éminents auteurs.

En ce qui concerne le corpus du travail, nous proposerons un bref aperçu de l'histoire des pièces de théâtre européennes adaptées ou traduites et représentées en Iran, pour ensuite nous mettre à déchiffrer la carrière d'Ali Raffi. Il est à noter que la mise en scène en Iran de ces pièces, à l'époque où la prise de risque liée à la circulation des savoirs entre la France et l'Iran demandait beaucoup d'efforts, n'était pas facile pour Raffi.

\section{2) Histoire des représentations scéniques à la française en Iran}

La rédaction des pièces de théâtre iraniennes à l'européenne remonte au XIXe siècle. Les dramaturges de l'époque tentaient d'écrire des pièces mettant en scène les plus célèbres épopées persanes, tout en décrivant des épisodes divertissants adaptés à des pièces occidentales. Dès lors, des critiques et des études comparatives entre les mythes orientaux et occidentaux ont vu le jour, telles des études comparatives entre le talon d'Achille et l'œil d'Esfandiâr'; entre l'aigle nommé Stanphal ${ }^{5}$ et l'oiseau intitulé Simorgh ${ }^{6}$; entre le Roi de la

${ }^{4}$ Le talon d'Achille (Mythe grecque) et l'œil d'Esfandiâr (Mythe persan) désignent le point faible de tout être. La seule partie vulnérable du corps d'Achille était son talon et celle d'Esfandiâr, son œil.

${ }^{5}$ Selon la mythologie gréco-romaine, Prométhée, étant contre la décision de Zeus à propos de la fondation d'une nouvelle race humaine. Il a volé un peu de feu de son oncle Volcänus 
Perse Cyaxases (Hovakhshatra en persan) et le Roi lydien Alyatte II, entre les mythes zoroastriens ${ }^{7}$ et les mythes gréco-romains.

Jusqu'à la moitié du XIXe siècle, plusieurs représentations théâtrales sans être inspirées des pièces occidentales se réalisaient en Iran, se focalisant plutôt sur des thèmes sociologiques, politiques et religieux inspirés de l'Iran même. À titre d'exemple, en 1850, Fath'Ali Akhoundzâdeh, rédigea une pièce théâtrale connue sous le nom de L'histoire de l'alchimiste Mollâ Ebrâbim Khalil. Cette pièce représente un hypocrite qui trompe les gens en prétendant qu'il est alchimiste et qui peut transformer le cuivre en argent. Il garantit certains d'entre eux qu'à force de travailler encore plus, il serait capable de leur rendre deux fois plus d'argent avec la même quantité de cuivre. Finalement, il s'est enfui avec toutes sommes reçues de la part de ce public superstitieux. Il fallut attendre une vingtaine d'années pour que Mirzâ Ja'far Gharatchedâghi la traduise de l'azéri vers le persan. Puis, cette œuvre, ainsi que d'autres comédies satiriques de ce même auteur furent traduites du persan vers d'autres langues européennes.

En outre, le premier secrétaire de l'ambassade de France à Téhéran, Mirzâ Aghâ Tabrizi publia, en 1871, des pièces de théâtre telles: Le voyage du Shâh Gholi Mirzâa à Kerbelâ, Les aventures d'Ashraf Khân à Téhéran, Les amours d'Aghâ Hâshem Khalkhâli, et Le mode de direction gouvernementale de Samsâm Khân de Boroujerd. Ses pièces étaient les signes d'une vision critique sur la société iranienne de son époque, et revêtent un caractère ironique. Puis, en 1886, sous le règne de Nassereddin Shâh Ghâdjâr (1831-1896), une première salle de théâtre iranienne à l'européenne destinée à la famille royale a été construite à l'École Polytechnique de Téhéran (Dâr Ol- Fonûn). Vu l'importance qu'accordait cette école à l'enseignement de la langue et de la littérature françaises, de nombreux traducteurs imprégnés de la richesse et de la

(Héphaïstos, Dieu du feu), et l'a donné aux hommes pour qu'ils ne soient pas abolis. C'est pourquoi, il a été condamné à être torturé par un aigle qui s'appelait Stanphal. Chaque matin, cet aigle lui dévorait le foie durant toute la journée et le soir, son foie se régénérait sans cesse et ce châtiment devrait se répéter pour l'éternité.

6 En persan, "Si" signifie "trente" et "morgh" équivaut "oiseau". Simorgh est un terme symbolique désignant les derniers trente oiseaux restant de ceux qui avaient décidé de parcourir le monde à la recherche d'un oiseau parfait qui s'appelait "Simorgh". Les uns sont morts, les autres, las de suivre le groupe, ont mis fin à leurs recherches et finalement ne restèrent que trente oiseaux qui, une fois unis, ont formé le "Simorgh" même. Cette histoire dont l'auteur est un poète, écrivain et philosophe iranien nommé Attâr, nous révèle que tout ce que nous cherchons à l'extérieur de nous, se trouve dans les profondeurs de notre monde intérieur.

7 "Ahura Mazda" qui désigne Dieu en Zoroastrisme [religion monothéiste née sur le territoire

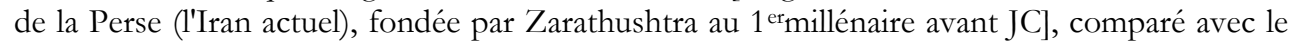
mythe grec intitulé Zeus. 
délicatesse de cette langue ont été formés. En premier lieu, des adaptations des pièces de Molière, telles Le Misanthrope, ou Le Médecin malgré lui, furent connues par le public. La traduction du Misanthrope de Molière y a été mise en scène en 1889. Puis, des adaptations de cette même pièce par des metteurs en scène tels Mirzâ Habib-é Esfahani, Mirzâ Fath Ali Khân Âkhoundzâdeh, Mirzâ Aghâ Tabrizi, et Morteza Gholi Khân Fekri Ershâd, ont connu un immense succès auprès du public.

Il arrive même à Mirza Habib-é Esfahani de traduire les paroles d'Alceste ${ }^{8}$ en se référant à un poème de Hafez (Fallahnejad, 93). Puis, cette même pièce a eu l'occasion d'être représentée sans aucune adaptation par une troupe théâtrale européenne à Téhéran. Les spectateurs iraniens se réjouissaient à distinguer les points communs et les différences qu'ils pressentaient entre les Européens et les Iraniens à travers les personnages des pièces de Molière. En 1888, un Club théâtral s'est formé au sein du quartier des Arméniens d'Ispahan. Il fallut attendre une dizaine d'années pour que les femmes fussent autorisées à jouer sur scène. Auparavant, les hommes se déguisaient en femmes pour pouvoir jouer les rôles assignés. Vu les interdictions religieuses, les femmes musulmanes étaient obligées de porter un voile et n'étaient pas autorisées à joindre d'autres acteurs masculins sur scène. Étant donné que les femmes chrétiennes avaient plus de liberté d'agir, les premières actrices à Ispahan furent alors trois jeunes filles arméniennes'. À cette même époque, Le Médecin malgré lui de Molière, ainsi que des pièces de Shakespeare tels Hamlet ou même Othello ont été représentées sur scène à Ispahan (Koushan 84).

Sous le règne de Reza Shâh Pahlavi (1925-1944), s’était également épanoui un théâtre nationaliste, se référant à la Perse antique. Dès lors, plusieurs pièces représentaient les incompréhensions naissant d'une confrontation entre les cultures occidentales et orientales, en dépit des échanges culturels entre l'Orient et l'Occident depuis l'Antiquité. Puis, la comédie en Iran fut développée par des metteurs en scène tels Ali Nasr et Esmaeil Mehrtâsh.

À l'époque de Mohammadreza Shâh (1941-1980), les pièces de théâtre ont adopté une dimension socio-politique. En effet, de retour vers le pays natal, les anciens étudiants en études théâtrales et cinématographiques des universités, des Grandes Écoles et des conservatoires européens se sont mis à traduire les pièces de théâtre européennes de diverses langues étrangères vers le persan. Il est à noter que pour des raisons politiques, il y a eu des fermetures temporaires

\footnotetext{
${ }^{8}$ Le personnage principal de l'œuvre de Molière intitulée Le Misanthrope, qui tombe amoureux de Célimène.

${ }^{9}$ Elles se nommaient Ossana Simonian, Zaghik Minassian et Lesabre Derhakoupian.
} 
de salles de théâtre. Vint ensuite la Révolution islamique et depuis, le théâtre a vécu plusieurs périodes de détentes et de tensions, quoique depuis 1997, une autorisation de mise en scène des pièces de théâtre ait été de nouveau émise, et les salles de théâtre ont été pour une nouvelle fois fréquentées, en particulier par les jeunes.

\section{3) Ali Raffi, des études sociologiques aux études théâtrales}

Ali Raffi, acteur, réalisateur et metteur en scène de théâtre iranien, né le 12 janvier 1939 à Ispahan (Iran), ville historique et touristique de l'Iran considérée comme un musée multiculturel, a poursuivi ses études primaires et secondaires au sein de sa ville natale. Gardien de but de l'équipe de football Shâhin lors de son adolescence à Ispahan, il a été lauréat d'une bourse sportive de la part du gouvernement iranien en 1957. Il a décidé de partir en France pour continuer ses études supérieures dans le domaine de la sociologie. Deux ans plus tard, en 1959, suite à une fracture du pied lors d'un accident de ski, sa vie a connu un changement remarquable qui lui a fait réaliser l'importance et la brièveté de la vie.

Titulaire d'une licence et d'un Master en sciences sociales de l'Université de la Sorbonne, il s'est intéressé au jeu d'acteurs au cinéma et au théâtre. Dès le début de sa carrière cinématographique, s'entraînant et participant à la réalisation des spectacles, il a pris conscience du rapport indissociable existant entre une vraie mise en scène cinématographique ou théâtrale et le jeu d'acteurs. C'est pourquoi il a eu envie de découvrir et de pratiquer les techniques du théâtre.

Pour mieux connaître ce metteur en scène et dramaturge iranien, il faut tout d'abord se référer à une période féconde $\mathrm{du} \mathrm{XX}^{\mathrm{e}}$ siècle qui était preuve du désarroi de l'homme dans une société en pleine mutation; une société qui avait déjà vécu l'entre-deux-guerres, la fermeture des salles de théâtre en 1918, et la censure des pièces de théâtre sous l'occupation allemande des années 40.

Les inquiétudes historiques des auteurs français de cette deuxième moitié du $\mathrm{XX}^{\mathrm{e}}$ siècle, issues d'un monde marqué par la cruauté et l'absurdité des deux guerres mondiales et la responsabilité et l'engagement de ces derniers vis-à-vis de la misère, l'injustice, le meurtre et la guerre, ont favorisé une sorte de débat tragique des hommes confrontant l'imaginaire et l'utilitarisme.

Les pièces de théâtre françaises du $\mathrm{XX}^{\mathrm{e}}$ siècle qu'a connues Raffi réfléchissent cette situation de l'être humain écartelé entre son idéalisme 
intransigeant et le réalisme conformiste, oscillant entre un sentiment de révolte et une acceptation de ses limites (Evrard 23).

Le Cartel des quatre directeurs de théâtre les plus connus de l'époque fondé en 1927, Gaston Baty (Studio des Champs-Élysées), Charles Dullin (Théâtre de l'Atelier), Louis Jouvet (Comédie des Champs-Élysées) et Georges Pitoëff (Théâtre des Mathurins) a pu s'épanouir. C'est à l'Atelier de Charles Dullin qu'Ali Raffi s'est initié vers 1962-1963, et a débuté sa carrière artistique par la pratique du théâtre.

À l'époque, l'Académie Charles-Dullin à Paris était le centre de présentation des ateliers du jeu d'acteurs, des cours de théâtre, des cours sur la direction d'acteurs, l'espace scénique, la mise en scène, la lecture d'une pièce de théâtre, les stratégies de conception et de direction d'un projet théâtral, ainsi que la considération du spectateur depuis son arrivée jusqu’à sa sortie de la salle.

Il est à noter que de grands acteurs tels Jean Marais, Marcel Marceau, Jean Vilar, Jean-Louis Barrault, Alain Cuny, Madeleine Robinson ont suivi une partie de leur parcours universitaire à l'école Charles Dullin. Celui-ci revendiquait l'autonomie de l'art de la mise en scène, qui avait une place prépondérante à l'époque. Concevant le théâtre comme un spectacle total où interviennent la danse, la musique et la pantomime, Dullin cherche à créer une émotion poétique qui lui paraît plus essentielle que la stricte expression de la réalité (Evrard 25).

Lors d'un entretien téléphonique, Raffi nous a révélé qu'à l'époque, l'Atelier de Charles Dullin était en rapport direct avec le TNP (Théâtre National Populaire), l'institution théâtrale fondée par Firmin Gémier en 1920, et située à Paris. Ce centre de théâtre a permis à toute une génération de prendre part à des spectacles de qualité. Dû à la succession des directeurs tels Albert Fourtier, Paul Abram, Pierre Aldebert, le TNP, visant un public populaire au départ, il s'est dédié à une élite par la suite. Puis, le TNP a été transféré de Paris à Lyon et a été dirigé par Jean Vilar qui réclamait un «théâtre pauvre » dépouillé du « décor » par souci de précision et pour faire plus avec moins sans se pencher sur « les spectateurs».

Les premières étapes du parcours artistique et culturel d'Ali Raffi ont eu lieu au théâtre. Titulaire d'un Master II en sociologie de l'Université de la Sorbonne, il a assumé le poste d'assistant à la mise en scène au Théâtre National Populaire (TNP) en France. 
Il lui a juste suffi de deux ans pour obtenir la position d'assistant au métier de metteur en scène de troisième degré au TNP, toujours en relation directe avec l'Atelier de Charles Dullin.

Il a bien compris la vision et l'orientation artistiques des metteurs en scène, a ajouté les annotations aux textes, a su maitriser le calendrier des répétitions et la rédaction des cahiers de régie, a bien minuté les scènes, et a agi d'une manière impeccable à titre d'intermédiaire entre les différents intervenants. Ce bras droit du metteur en scène qu'était Raffi a franchi le grade d'assistant au métier de metteur en scène de deuxième degré, et puis de premier degré en deux ans pour chaque étape. Durant onze ans, il a travaillé au sein du Théâtre National Populaire, sous la direction de Georges Wilson.

Ce n'est qu'après l'obtention du poste d'assistant au métier de metteur en scène de premier degré qu'il a pressenti la nécessité d'une solide formation académique et s'est inscrit à l'Institut des Études théâtrales de l'Université de la Sorbonne Nouvelle- Paris 3, Censier. C'est là qu'il a fait la connaissance du professeur Bernard Dort (1929-1994), qui a dirigé sa thèse de doctorat, en plus de devenir un ami, un maitre et un initiateur qui lui a révélé les secrets des arts du spectacle. Durant les dix années de coopération et d'assistanat auprès de Dort qui était titulaire de poste de professeur en Esthétique et Sciences de l'art, Ali Raffi a eu l'occasion de raffiner ses idées et de revoir sa conception du théâtre.

Directeur du Théâtre et des Spectacles au Ministère de la Culture, Dort a été membre du Comité de rédaction de la revue Théâtre populaire depuis sa création (1953-1964), de la revue Travail Théatral (1970-1980), et membre du Comité de direction de la Revue Théâtre en Europe. Cette figure centrale de la critique dramatique contemporaine a formé toute une génération à la pensée de Bertolt Brecht, sur qui il a écrit sa thèse de doctorat. Il a également supervisé des travaux sur Jean Genet et Arthur Adamov, dramaturge francoarménien influencé par le surréalisme. Raffi a fait sien les centres d'intérêt de son maitre.

Titulaire d'un doctorat en Études théâtrales, de l'Université de la SorbonneNouvelle à Paris, Raffi a rédigé une thèse de doctorat intitulée Les théatres hors théâtre en France de 1960 à 1973 (1974), sous la direction de Bernard Dort. Raffi était également influencé par les œuvres des metteurs en scène les plus connus du $\mathrm{XX}^{\mathrm{e}}$ siècle, tels Meyerhold, Constantin Stanislavski, Jacques Lecoq, et Giorgio Strehler, Bertolt Brecht, Federico Garcia Lorca, Jacques Copeau, Jean Genet, et André Antoine connu comme le père de la mise en scène moderne. Pendant un certain temps, Ali Raffi a travaillé avec Jacques Lecoq, metteur en 
scène français. Lecoq était considéré comme l'un des plus grands spécialistes de mimes dramatiques et des chœurs de tragédies antiques. Il a eu l'occasion d'observer de près une approche mimo-dynamique des arts et du théâtre, de discerner les divers techniques appliquées aux différents styles dramatiques, de prendre part aux stages organisés par ce dernier à l'École Internationale du Théâtre Jacques Lecoq. Puis, Raffi a décidé de quitter Paris en 1974 pour retourner dans son pays natal, et commencer une carrière universitaire au sein de l'Université des Beaux-Arts de Téhéran. De retour en Iran, plusieurs postes administratifs lui ont été offerts, mais, il s'est toujours focalisé sur la scène et l'enseignement.

\section{4) Raffi inspiré par la dramaturgie classique européenne, plus particulièrement française}

Ali Raffi, partisan de l'esthétisme, a mis de côté les conventions scéniques mises en pratique en Iran, et a proposé au public une conception théâtrale enrichie. En effet, comme le dit Charles Mauron dans Psychocritique du genre comique, «la réalité extérieure et l'imaginaire alimentent dans des proportions variables toute création esthétique »(25). Mais, vu les problèmes sociopolitiques de l'époque en Iran, il n'était pas si facile pour Raffi de mettre de côté la réalité extérieure, quoique grâce à la force de l'imaginaire, il a su alimenter sa création esthétique.

Juste un an après son retour en Iran, Raffi a su organiser une pièce théâtrale estudiantine par la mise en scène d'Antigone, au Salon de théâtre Mowlavi de Téhéran. Il a su adapter et mettre en scène Crime et Châtiment ${ }^{10}$ de Fiodor Dostoïevski, en Iran en 1977. Inspiré par Dort, Raffi à son retour en Iran, a également mis en scène Les Bonnes de Jean Genet (en 2002) au Théâtre de la ville de Téhéran (City Theater of Tehran, Théatr-é Šabr-é Tehrân)- Salle Tchâhârsû.

Pour Dort, l'approche pratique et la réflexion théorique allaient de pair. Auteur de divers ouvrages tels : Théâtre/Public (1967); Théâtre réel (1971); Théâtre en jeu (1979); La Représentation émancipée (1988); ainsi que Le Spectateur en dialogue (1995), Dort a reflété le paysage théâtral français depuis le début des années cinquante. Ainsi, il a su purifier et rectifier la pensée théâtrale de ses disciples, et dans le cadre du Théâtre populaire, il a fait découvrir à la France, le théâtre épique de Bertolt Brecht. Il était connu pour ses analyses de spectacles et d'œuvres théâtrales.

${ }^{10}$ En persan, ce livre a été traduit sous le titre de Jénâyât-O-Mokâfât, ce qui désigne Crimes et Châtiments. 
Ali Raffi ${ }^{11}$ nous dévoile que pour mieux saisir la délicatesse, la lucidité et la sagacité d'esprit de Dort, il suffit de mentionner que d'après ce maittre, pour être imbibé de sagesse, de clairvoyance et de perspicacité dans le domaine, il faut se référer aux sources initiatrices et créatrices anciennes et classiques au lieu de consulter les imitations.

Selon Raffi, la majorité des œuvres classiques sont immortelles. Autrement dit, une nouvelle représentation scénique et une nouvelle lecture de la majorité de ces œuvres sont productives et fertiles :

En vers ou en prose, sur le fond ou la forme, ces œuvres nous communiquent d'un langage poétique et nous influencent d'un pouvoir magique et incontestable. Le secret de l'immortalité de ces chefs-d'œuvre consiste au fait que malgré l'ancienneté et les divers évènements qui se sont effectués depuis leur création sur ce globe terrestre, ils nous paraissent toujours originaux, récents, innovants, et nous permettent de découvrir des nouveautés au sein de leur propre monde intérieur (2).

Dort était contre le dogmatisme et le formalisme; il croyait fermement que l'unité de base n'était ni la pièce et son auteur, ni la performance de tel ou tel acteur, mais la représentation. Il supposait qu'écrire sur la représentation, c'était prolonger le dialogue entre l'œuvre et le monde en interrogeant la responsabilité sociale, et pas seulement littéraire, du théâtre (Porée 205).

C'est la raison pour laquelle, dès le début de travail de Raffi en Iran, l'esthétisme classique était l'élément le plus représentatif de ses scènes (Shahbazi 8). Ensuite vinrent les années où Ali Raffi, selon les recommandations de Bernard Dort, a eu l'occasion de se familiariser avec les œuvres de Vsevolod Emilievitch Meyerhold, dramaturge et metteur en scène russe, qui était Directeur du Théâtre de la révolution en 1922 et fut victime des purges staliniennes une fois emprisonné.

Le système de formation d'acteur développé par Vsevolod Meyerhold porté le nom de «biomécanique » ${ }^{12}$. Le but de Meyerhold était d'élargir le potentiel émotionnel d'une pièce de théâtre afin de pouvoir mieux exprimer ses pensées et ses idées, ce qui manquait au théâtre naturaliste et réaliste de l'époque. Ces techniques de biomécanique, incompatibles avec le réalisme socialiste russe qui se contentait de son apogée en Russie, eurent ensuite, une grande influence sur le théâtre contemporain.

11 Lors d'une interview téléphonique le 10 juin 2019, j'ai eu l'occasion de lui poser des questions et il m'a autorisée à traduire ses réponses pour des publications ultérieures.

${ }^{12}$ La biomécanique est une discipline qui associe la "biologie" et la "mécanique". Elle étudie la reproduction des mécanismes qui déterminent les mouvements de l'acteur sur scène. Ainsi, le corps de l'acteur devient un véhicule d'action qui a une certaine similitude à une machine. 
Le décor ne comptait point pour Meyerhold; au contraire, il préférait des costumes simples qui permettaient un entraînement physique réussi lors des danses et des comédies musicales. Cet ancien élève de Konstantin Stanislavski du Théâtre d'art de Moscou et metteur en scène d'avant-garde, était contre le réalisme et le psychologisme et envisageait un théâtre qui n'imite pas la vie. Il ne donnait pas d'importance aux conventions ordinaires :

Convention, qu'est-ce qu'à dire?

Il ne s'agit pas bien sûr, de la convention routinière, odéonesque, non avouée, inconsciente même...mais d'une convention consciente, revendiquée, réfléchie : jeu de thêâtre mené selon des règles admises par acteurs et spectateurs (Porée 228).

Lors d'un entretien effectué par Bahareh Borhani, intitulé "Souvenirs et Dangers vécus par Monsieur l'Artiste », publié dans la Revue Paragraphe, Ali Raffi avoue que pour lui, la grande révolution théâtrale du XXe siècle est due aux travaux de trois grands dramaturges : Edward Gordon Craig (1872-1966), Adolf Appia (1862-1928) et Vsevolod Meyerhold (1874-1940). Raffi y mentionne également que ces trois personnes ont toujours été en quête de nouvelles techniques pour renouveler l'esthétique théâtrale et libérer le théâtre de ses problèmes emblématiques ${ }^{13}$, de toute tyrannie ${ }^{14}$.

Accordant une place primordiale à l'esthétisme, Raffi a également mis en scène des pièces avec des couleurs attrayantes ${ }^{15}$ et une élégance appréciée par les spectateurs. Pourtant, pour revenir à Meyerhold, nous avons recours à Alexandre Taïrov, l'un de ses élèves, qui prend position et attaque Edward Gordon Craig pour sa conception de la surmarionnette, qui sert à décrire le jeu de l'acteur tel qu'il l'imaginait (Veinstein) et Meyerhold pour avoir prôné un théâtre de la convention consciente ${ }^{16}$, art de la représentation qu'il juge trop formel (Taïrov 212)

Marie-Dominique Porée explique que Meyerhold orientait ses acteurs athlètes et ses comédiens vers des jeux basés sur un système de signes élitaire ${ }^{17}$,

13 Telle la distribution des rôles au cas où tous les acteurs auraient le même âge, la même ancienneté et la même habileté professionnelle.

${ }^{14}$ Lors des clowneries, par exemple, «le rire est souvent interrompu par des discussions qui font apparaître les difficultés à dénoncer la tyrannie» (Bradby 307).

${ }^{15}$ Raffi utilise des couleurs qui, se caractérisant par leur beauté et leur aspect agréable. À titre d'exemple, pour éclaircir ou assombrir une scène, il fait intervenir le blanc et le noir, et pour donner plus de chaleur il se sert d'un rouge vif.

${ }^{16}$ Le théâtre de la convention consciente anéantit la vulgarité théâtrale par des procédés de théâtralité et aboutit au vrai théâtre.

${ }^{17}$ Cette méthode biomécanique exige que les acteurs se concentrent sur des systèmes de signes non-verbaux (langage du corps comprenant les mouvements faciaux et les autres formes 
déjà connu du public. Elle affirme de même que Meyerhold ne valorise ni le décor, ni la splendeur des costumes qui paralysent les gestes de l'acteur. Pour lui, l'acteur est tout. Les décors encombrants tels les escaliers et les praticables du décorateur Appia tout comme les rideaux de Craig n'ont plus de raison d'être chez lui. Seul compte le travail gestuel, qu'il soit individuel ou collectif d'ailleurs, des comédiens acrobates et gymnastes qui suivent, à la virgule près, une méthode quasi biomécanique (Porée 228).

Avec Raffi, nous avons recours à cette même idéologie :

Nous n'avons pas besoin de mettre en scène une maison telle qu'elle est avec tout son ameublement pour engendrer la notion de maison chez le spectateur, à la manière des naturalistes tels André Antoine et le Russe Constantin Stanislavski. [...] Appia pense qu'il suffit de créer un espace et transformer cet espace en lieu désiré par les gestes, les actes et les jeux de rôle des acteurs. Craig et Meyerhold aussi le disent de leur propre manière. (Borhani 18).

Plus loin Raffi ajoute que :

Nous n'avons pas besoin de créer des décors qui incarnent la notion de forêt pour engendrer cette notion chez le spectateur. Il suffit d'avoir un espace vide et de demander aux acteurs de faire semblant de jouer dans une forêt. L'espace est le même; c'est l'acteur qui le transforme en château, salle de bain, atelier, usine, ou autres (19).

Malgré les germanophobies de certains Français et les réticences issues des souvenirs de l'Occupation, suite aux recommandations de Bernard Dort qui s'intéressait autant à la pensée fondamentale occidentale qu'orientale, à l'époque où le «Mur de Berlin » séparait les deux Berlin-Est et Berlin-Ouest, Ali Raffi s'est également instruit sur Berliner Ensemble, l'une des plus grandes troupes de la deuxième moitié du XXe siècle. En fait, Raffi s'était inspiré d'un théâtre fondé à Berlin-Est par Bertolt Brecht. Brecht qui a toujours été en quête d'une osmose entre didactique et divertissement, nous rappelle que la tâche assignée à la tragédie par les Anciens n’est ni plus ni moins que de divertir les hommes (Porée 146). Par ailleurs, il avoue qu'il garde pour lui toute liberté de prendre plaisir à instruire (146).

Avec la théorie de "distanciation ${ }^{18}$, Brecht rejette toute identification de son acteur avec le personnage. Se référant à l'usage des masques pendant

d'expressions corporelles) et verbaux, convenables à une élite intellectuelle. De plus, les mouvements corporels importaient beaucoup pour Meyerhold.

18 En se référant à la théorie de Brecht, pour un metteur en scène, la distanciation est une méthode qui vise à créer une certaine distance entre le spectacle et le spectateur, par le choix du sujet, certaines techniques de mise en scène, par le jeu des acteurs, en vue de développer l'esprit critique de l'audience. « L'avantage essentiel que le théâtre épique tire de la distanciation (laquelle vise exclusivement à montrer le monde sous un angle tel qu'il apparaisse comme susceptible d'être pris en main par les hommes), c'est justement son caractère naturel et 
l'Antiquité qui créaient une distance entre les acteurs et les spectateurs, il déclare dans son Petit Organon pour le théâtre:

Une reproduction qui distancie est une reproduction qui, certes, fait reconnaittre l'objet, mais qui le fait en même temps paraitre étranger. Les théâtres antiques et médiéval distanciaient leurs personnages avec des masques humains et animaux, le théâtre asiatique utilise aujourd'hui encore des effets de distanciation musicaux et pantomimiques (40).

Marie-Dominique Porée affirme de même que «pour Brecht, le spectateur et le comédien devraient non pas se rapprocher, mais au contraire s'éloigner l'un de l'autre. Autrement dit, chacun devrait s'éloigner de soi-même. Le spectateur dépose au vestiaire sa raison en même temps que son manteau » (184). Par contre, comme cite Alain Badiou ${ }^{19}$ dans l'ouvrage intitulé Éloge du Théâtre, Jacques Rancière se demande si ce n'est pas justement «la volonté de supprimer la distance qui crée la distance » (38).

Cette distance entre les acteurs et les spectateurs est également à saisir lors des pièces de Raffi, puisque les spectateurs iraniens ignorant la mentalité et les systèmes de signes verbaux et non-verbaux occidentaux, trouvent une certaine distance avec les acteurs du point de vue moral, mais également du point de vue physique, puisque Raffi a l'habitude de choisir des salles de représentation assez grandes.

Pour ce qui est de la mise en scène de la pièce de théâtre Antigone, Raffi s'est inspiré à la fois de Jean Anouilh, de Brecht et de Sophocle (20) ${ }^{20}$. Brecht a mis en scène l'Antigone de Sophocle en 1947-1948. Il n'a point voulu recréer une pièce déjà créée par Sophocle, comme Raffi d'ailleurs. Selon Maurice Regnaut, traducteur de l'Antigone de Bertolt Brecht, " l'Antigone que Brecht tire de Sophocle via la traduction de Hölderlin est un exemple magnifique de son art de la copie, fidèle dans le style, différent dans le dessein » (3).

Dort avait également présenté à Raffi, Jacques Lecoq, et Giorgio Strehler, l'un des plus célèbres metteurs en scène italiens, partisan de la commedia dell'arte. C'est la raison pour laquelle, vu que Giorgio Strehler avait mis en scène le 26 février 1948, Crime et Châtiment de Fiodor Dostoïevski; Raffi a mis

terrestre, son humour, son refus de toute cette mystique dont le théâtre traditionnel est redevable à des époques depuis longtemps révolues » (Brecht 337).

${ }^{19}$ L'ouvrage représente un dialogue public entre Alain Badiou et Nicholas Truong, qui a eu lieu le 15 juillet .2012, dans le cadre du «Théâtre des idées», cycle de rencontres intellectuelles et philosophiques du Festival d'Avignon.

20 Raffi s'est inspiré de Jean Anouilh puisqu'il a voulu produire une représentation contemporaine d'Antigone; il s'est inspiré de Sophocle pour le thème d'Antigone, et de Brecht pour la représentation de « l'effet de distanciation ». 
en scène ladite pièce. Raffi a mentionné que pour toute pièce occidentale, mais non française, il s'est toujours référé à la traduction française pour les œuvres qui n'étaient pas encore traduites ou à la traduction persane des œuvres déjà traduites, puisqu'il n'avait pas le temps de retraduire les pièces d'une langue autre que le persan vers cette langue et la mettre à la disposition de ses acteurs. Giorgio Strehler avait également mis en scène le 21 avril 1955, La Maison de Bernarda Alba, de Federico Garcia Lorca, poète, dramaturge, pianiste, compositeur, peintre et prosateur espagnol.

Raffi a mis en scène cette même pièce durant l'hiver 2019, alors qu'on lui fêtait son quatre-vingtième anniversaire à Téhéran. La foule fut nombreuse et motivée. Pour cette pièce, Raffi s'est référé à la traduction persane d'Ahmad Shâmlou.

Se référant aux mises en scène de Giorgio Strehler recommandées par Dort, Raffi a réussi à mettre en scène trois pièces de Federico Garcia Lorca traduites de l'espagnol ou du français vers le persan par Ahmad Shâmlou. Cela s'explique par le fait que les jeux de sonorités, les idées ingénieuses pour trouver des termes clés dans les traductions, et les trouvailles sémantiques inattendues qu'introduisait Shâmlou (Ayati et Mahdavi) lui plaisaient beaucoup.

Les trois pièces susmentionnées et mises en scène par Raffi en Iran étaient : Yerma (2013), pièce poétique symbolique qui présente le conflit intérieur d'une paysanne mariée qui attend désespérément de tomber enceinte; La Maison de Bernarda Alba (2019), drame en trois actes, montrant le rôle secondaire occupé par la femme dans l'Espagne rurale du début du XXe siècle, malgré le caractère dominateur de Bernarda; et Noces de sang (1998-1999), drame qui retrace l'histoire tragique d'une passion impossible, mais irrésistible, au sein d'une société traditionnelle en Andalousie, avec une approche géo-poétique, sans toutefois mettre de côté les liens entretenus entre l'auteur et le fantastique issu du surréalisme.

En ce qui concerne les autres mises en scène de Raffi, il est à noter que sa pièce intitulée La Chasse au renard, mise en scène en 2008 au Théâtre Vahdat de la ville de Téhéran, pourrait être une inspiration de La Chasse au renard, écrite par M. de Saint- Hilaire. Dans son abrégé intitulé The House of Bernarda Alba, Raffi annonce que les grands metteurs en scène croient que tout spectacle qui n'est pas une réponse à un problème social, ne vaut pas la peine d'être représenté sur scène (2), et La Chasse au renard de Raffi répond à cette obligation.

\section{Conclusion}


Certains metteurs en scène préfèrent supprimer tout ce qui suggère le passé, d'autres non. Certaines personnes qui dirigent la représentation d'une œuvre ou la réalisation d'un film comme Jean Vilar, vident l'espace scénique pour offrir une version plus épurée; d'autres comme Bertolt Brecht immergent les acteurs dans un espace encerclé par des spectateurs. Plus précisément, Vilar et encore plus Peter Brooke réclamaient un "théâtre pauvre" dépouillé du "décor" par souci de précision et pour faire plus avec le moins sans se pencher sur "les spectateurs" alors que Brecht ne se penche pas sur le dépouillement du décor en tant que tel, mais revendique une "distanciation" sur le plan du jeu de l'acteur. Pour Ali Raffi, ni le passé ni le présent, ni le vide ni l’immensité de la foule ne comptent; ce qui l'intéresse surtout c'est l'esthétisme et le culte du beau. Raffi a toujours été en quête de ce qui se trouvait à l'intérieur de la conscience. À l'image de son éminent professeur et ami Bernard Dort, et après avoir vécu une période assez féconde en France, il est retourné au pays natal, suite à la découverte de Meyerhold, Bertolt Brecht, Jacques Lecoq, Edward Gordon Craig, Adolf Appia, Federico Garcia Lorca, Jean Genet, Adamov, et Giorgio Strehler. Il a su mettre en scène et adapter des pièces de théâtre européennes qu'il jugeait plus pertinentes, dotées de bon sens, de la délicatesse et de la justesse d'esprit. Étant donné son patriotisme, il a tout fait pour éveiller l'activité intellectuelle de ses compatriotes, dans une période où les salles étaient plutôt vides et le public avait des exigences élevées en matière de mise en scène. Pour Raffi, l'imagination des sources théâtrales occidentales ou orientales avaient une place primordiale. Durant sa carrière éblouissante, Raffi a eu toujours tendance à sauvegarder le cadre et l'esthétisme classique pour reproduire l'âme intemporelle de ce genre théâtral présenté sous forme de pièces contemporaines qui ne dépendent pas des dimensions spatiotemporelles. Ses prises de position théâtrales ne sont absolument pas politiques, mais plutôt sociales. Ce réformateur intelligent et inventif a préconisé un art poétique simple, accompagné de musique et de rêve, sous forme d'images intemporelles inoubliables.

Ayant créé un atelier professionnel d'art dramatique, il a formé non seulement des centaines d'étudiants, mais aussi maints formateurs. Il a assumé un grand rôle pour la vivacité du milieu théâtral en Iran et le développement culturel du public. 
Alternative Francophone vol.2.5 (2020): 6-20

http://ejournals.library.ualberta.ca/index.php/af

\section{Bibliographie}

Ayati, Akram et Mahdavi Zadeh, Mojgan. «La réception de l'imagination poétique aragonienne, chez Ahmad Shâmlou, poète contemporain iranien ». Revue Alternatives Francophones, vol 2, no 2, 2018, pp. 78-92.

Badiou, Alain, et Truong Nicolas. Éloge du théâtre. Champs essais, 2016.

Borhani, Bahareh. "Souvenirs et Dangers vécus par Monsieur l'Artiste". Revue des Arts du spectacle Paragraphe, vol. 1, no 1, 2016, pp. 11-43.

Bradby, David. Le théâtre françis contemporain : 1940-1980. Presses Universitaires de Lille, 1990.

Brecht, Bertolt. Écrits sur le théâtre. Traduit par J. Tailleur, L'Arche, 1972.

---. Petit organon pour le théatre (Kleines Organon für das Theater). Traduit par Jean Tailleur, L'Arche, 2013.

---. Antigone (Die Antigone des Sophokles). Texte français par Maurice Regnaut, L'Arche, 2000.

Evrard, Franck. Le théâtre français du XXe siècle. Ellipses, 1995.

Fallahnejad, Naeimeh. "L'influence du théâtre français sur le théâtre persan moderne, à la fin du XIX ${ }^{\mathrm{e}}$ siècle : le rôle des traductions et des traducteurs". Alternatives Francophones, vol. 1, no 8, 2015, pp 86-99.

Koushan, Naser. Târikh-é théâtre dar Esfahân (The History of Theater in Esfahan). Atropat, 1379 (2000).

Mahdavi Zadeh, Mojgan. Phone conversation with Ali Raffi. 10 June 2019.

Mauron, Charles. Psychocritique du genre comique. Librairie José Corti, 1985.

Porée, Marie-Dominique. Le Théâtre pour Les Nuls. Éditions First \& Willey Publishing, 2014.

Raffi, Ali. The house of Bernarda Alba. Éditions Tcheshmeh \& Bonyad-é Shamlou, 2018-19.

Shahbazi, Ramtin. "L'Ésthétisme classique, et les raisons de l'importance d'Ali Raffi ». Revue des Arts du spectacle Paragraphe, vol. 1, n 1, 2016, pp. 8-9.

Stanislavski, Constantin. La formation de l'acteur. Translated from English by Élisabeth Janvier, preface by Jean Vilar, Petite Bibliothèque Payot, 2015.

Taïrov, Alexandre. Le Théatre libéré. L'Âge d'homme, 1974.

Veinstein, André. Craig Edward Gordon (1872-1966), Encyclopadia Universalis, http://www.universalis.fr/encyclopedie/edward-gordon-craig/. Accessed 29 December 2019. 